\title{
Diffuse Bacterial Meningitis and Myelitis Secondary to a Diplococcus Organism
}

\author{
Ghita Ann Wiebe, Sergio Fanella, Jens Wrogemann, Carrie Daymont, Mubeen Rafay
}

Key words: Meningitis, Pediatric Neurology

doi:10.1017/cjn.2015.317

Can J Neurol Sci. 2016; 43: 192-194

A 16-year-old, previously healthy, female presented with a 1-week history of sore throat, fever, vomiting, and occipital headache. She also complained of generalized myalgia and lower limb weakness. There was no medication or substance use. Her immunizations were up to date, and she had no recent travel history or contact with persons from overseas. Examination in emergency revealed an unwell girl with nuchal rigidity and generalized hyperesthesia. Muscle strength in the lower extremities was graded 3-/5. In the upper extremities, strength was graded $4+/ 5$ except distally on the right side, which was graded 4-/5 with scooping fingers and difficulty with extension of fingers, along with weak wrist flexion. Sitting was noted to be difficult. Reflexes were decreased in the lower limbs; brisk reflexes were found in the upper limbs.

Initial magnetic resonance imaging (MRI) showed extensive T2 signal changes throughout the spinal cord (Figure 1A,B). MRI of the brain did not reveal any significant intracranial abnormality. Initial differential diagnosis included demyelinating myelitis from an infectious, postinfectious, or inflammatory process. Infarction and neoplastic infiltration of the spinal cord were also considered. Her cerebrospinal fluid (CSF) had increased protein $(2.29 \mathrm{~g} / \mathrm{L})$, lactate $\left(4.2 \mathrm{mmol} / \mathrm{L}\right.$ ), cell count $840 \times 10^{6} / \mathrm{L}$ (neutrophils, 39; lymphocytes, 53; monocytes/macrophages, 8) and normal glucose $(2.5 \mathrm{mmol} / \mathrm{L})$. A CSF Gram stain revealed gram-negative diplococci versus coccobacilli (orientation of organism unclear). Blood and CSF cultures were obtained before treatment with intravenous cefotaxime and vancomycin at meningitic doses and dexamethasone ( $7.5 \mathrm{mg}$ every 6 hours).

Overnight, she developed progressive flaccid paralysis of extremities; cefotaxime was switched to ceftriaxone and dexamethasone was switched to daily methylprednisone $(1 \mathrm{~g}$ for 5 days) followed by oral prednisone $(1 \mathrm{~g} / \mathrm{kg})$ in tapering doses over 20 days. She demonstrated gradual improvement of her extremity weakness. Repeat MRI showed minimal interval improvement (Figure 2). CSF and blood cultures yielded negative results, as did CFS analysis using 16S RNA PCR. ${ }^{1}$ CFS, stool, and nasopharyngeal secretion viral cultures (including polio virus) revealed no causative organism. She was treated with 14 days of antibiotics for presumed bacterial meningitis resulting from either Neisseria meningitidis or Haemophilus influenzae, based on the initial CSF Gram stain. Follow-up MRI 1 month after presentation revealed improvements, with minor residual inflammation/demyelination seen within the cervical/thoracic spinal cord (Figure 3). Two months after her presentation, she was ambulating with cane assistance. A repeat MRI 9 months after presentation showed resolution of abnormal findings. Follow-up 11 months after presentation showed almost complete neurological recovery, with only mild residual bowel and bladder dysfunction.

\section{Comment}

Meningitis presenting with diffuse myelopathy is rare. Proposed mechanisms of spinal cord damage include infarction, autoimmune-mediated infectious/postinfectious inflammatory myelopathy, direct infection, vasculitis, and hypoxia. ${ }^{2-6,8}$

Myelitis, an inflammation of the spinal cord, is a rare complication of meningococcal meningitis that usually occurs during treatment or as a late complication. ${ }^{2-8}$ The thoracic cord and conus medullaris are most commonly affected. ${ }^{4}$ Symptoms may evolve over hours or days, with lower extremity flaccidity, bowel and bladder dysfunction, and a reduced sensory level. ${ }^{7}$ Reported MRI findings include intramedullary signal on T2-weighted images, cord swelling, leptomeningeal and intramedullary contrast enhancement. ${ }^{4-5,8}$ Enhancement of the cauda equina and lumbosacral nerve roots is also documented. ${ }^{8}$

Overall prognosis is guarded, with residual deficits including bowel/bladder dysfunction and ambulation difficulties. It is difficult to determine the contribution of steroids in neurological recovery. ${ }^{3,5-6,8}$ Since 1996, The Canadian Pediatric Surveillance Program has maintained a protocol to identify and investigate acute flaccid paralysis in children. ${ }^{9,10}$

In our patient, CSF pleocytosis and biochemical analysis with positive Gram stain confirms an infectious etiology. Possibilities for cultures being negative include artifact on Gram stain or accumulation of the stain. Another possibility is that there was failure of the polymerase chain reaction because of a dead organism. Either direct infectious or infectious immune-mediated mechanisms are suspected for this diffuse myelitis. This rare presentation highlights considerations for an acute infectious diffuse myelopathy in patients that present with acute-onset progressive extremity weakness.

From the Section of Child Development (GAW), Pediatric Infectious Diseases (SF), Department of Pediatric Radiology (JW) Children's Hospital Research Institute of Manitoba, (CD, MFR), Section of Pediatric Neurology, (MFR), Department of Pediatrics and Child Health, (GAW, SF, JW, CD, MFR), Children's Hospital Winnipeg, University of Manitoba. Received July 11, 2014. Final Revisions Submitted July 15, 2015.

Correspondence to: Ghita Ann Wiebe, Section of Child Development, Department of Pediatrics and Child Health, CK253 840 Sherbrook Street, Winnipeg,

MB R3A 1S1. Email: gwiebe4@exchange.hsc.mb.ca 

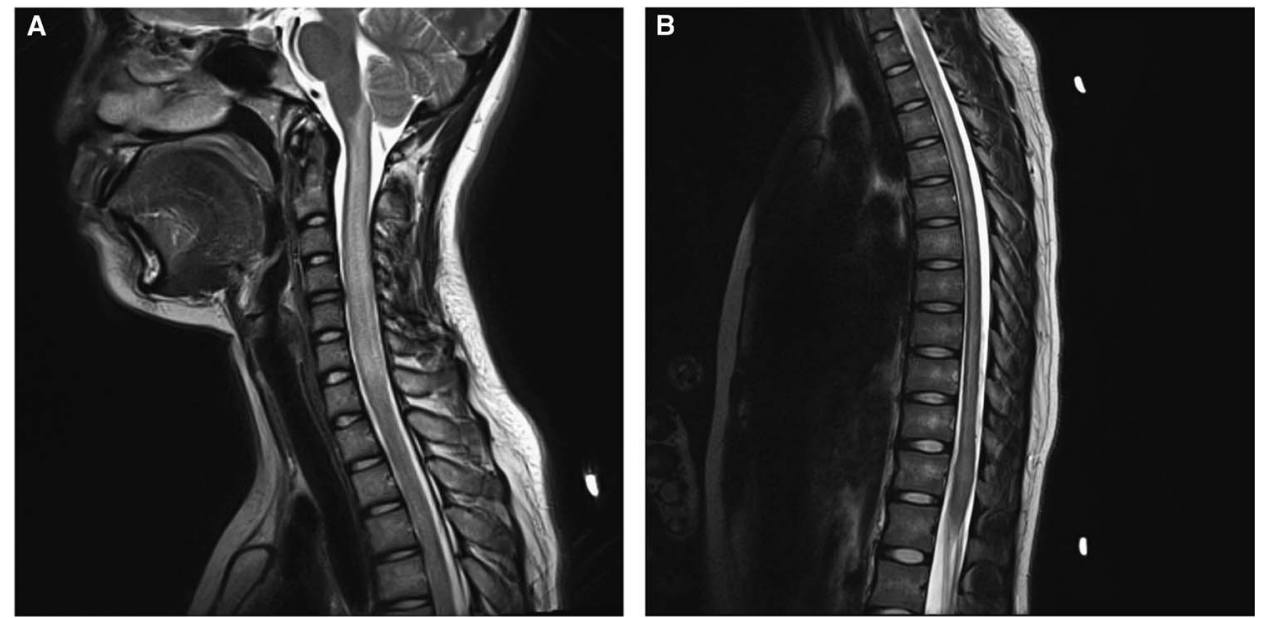

Figure 1: (A) MRI cervical spine sagittal T2 showing evidence of extensive high signal changes throughout the spinal cord starting in the lower medulla and involving preferentially the deep gray matter of the spinal cord. There is also swelling of the entire cervical spinal cord. (B) MRI thoracic spine sagittal T2 demonstrating evidence of extensive hyperintense signal changes in thoracic spinal cord.

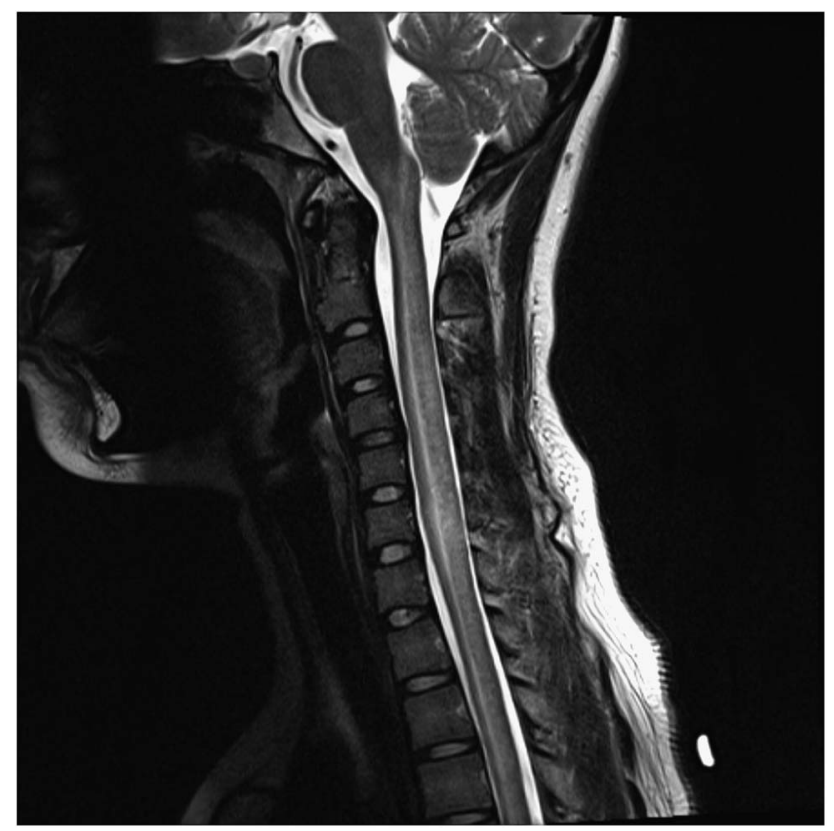

Figure 2: Repeat MRI cervical spine sagittal T2 done 5 days after presentation and treatment. Evidence of slightly less cord swelling and less signal abnormality is noted in the cervicothoracic spinal cord.

\section{Disclosures}

Ghita Ann Wiebe, Mubeen Rafay, Sergio Fanella, Jens Wrogemann, and Carrie Daymont have nothing to disclose.

There was no financial support or conflict of interest identified.

\section{REFERENCES}

1. Edwards U, Rogall T, Blöcker H, Emde M, Böttger EC. Isolation and direct complete nucleotide determination of entire genes: characterization of a gene coding for $16 \mathrm{~S}$ ribosomal RNA. Nucl Acids Res. 1989;17:7843-53.

2. Khan J, Altafullah I, Ishaq M. Spinal cord dysfunction complicating meningococcal meningitis. Postgrad Med J. 1990;66:302-3.

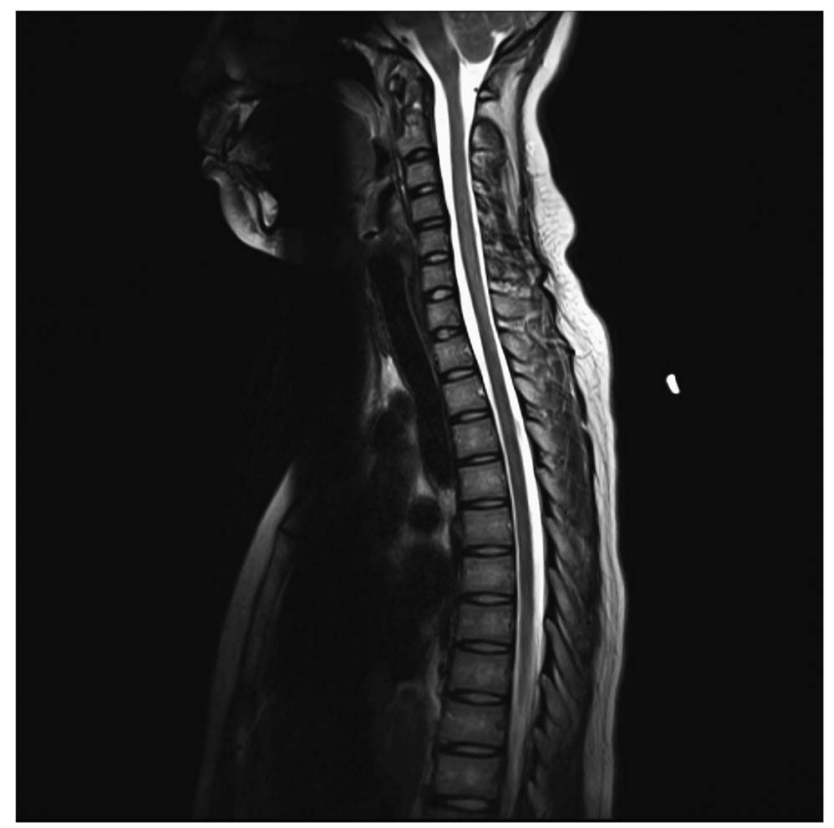

Figure 3: MRI cervical and upper thoracic spine sagittal T2 done 1 month after the patient's presentation reveals resolution of cord swelling and evidence of only minor residual hyperintense signal abnormalities.

3. Ibrahim WH, Elalamy OR, Doiphode SH, Mobyaed H, Darweesh A. Acute myelopathy with sudden paraplegia as the sole manifestation of meningococcal meningitis. Libyan J Med. 2010; 8:4862-4.

4. Bhojo AK, Akhter N, Bakshi R, Wasay M. Thoracic myelopathy complicating acute meningococcal meningitis: MRI findings. Am J Med Sci. 2002;323:263-5.

5. Choudhary N, Kumar N, Ahlawat R, Kapil Jain, Yasir Rizvi, Gaurav Agarwal, et al. Meningococcal meningitis with myelopathy: case report and review of literature. CE J Med. 2007;2:122-7.

6. Rathore MFA, Gill ZA, Malik AA. Acute flaccid paraplegia: a rare complication of meningococcal meningitis. Spinal Cord. 2008;46:314-6. 
7. Roos KL. Comment on: acute severe spinal cord dysfunction in bacterial meningitis in adults. MRI Findings suggest extensive myelitis. Arch Neurol. 2001;58:717-8.

8. Kastenbauer S, Winkler F, Fesl G, Schiel X, Ostermann H, Yousry TA, et al. Acute severe spinal cord dysfunction in bacterial meningitis in adults. Arch Neurol. 2001;58:806-10.
9. Desai S, [Internet]. Acute flaccid paralysis. Canadian Paediatric Surveillance Program. Available from: http://www.cpsp.cps.ca/ surveillance/study-etude/acute-flaccid-paralysis.

10. CPSP Highlights. Poliomyelitis: are Canadians still at risk? Pediatr Child Health. 2007;12:708. 